\title{
Liposomes encapsulating polymeric chitosan based vesicles a vesicle in vesicle system for drug delivery
}

\author{
Deborah McPhail a, Laurence Tetley ${ }^{\mathrm{b}}$, Christine Dufes ${ }^{\mathrm{a}}$, \\ Ijeoma F. Uchegbu ${ }^{a, *}$ \\ ${ }^{a}$ Department of Pharmaceutical Sciences, University of Strathclyde, Strathclyde Institute for Biomedical Sciences, 27 Taylor Street, \\ Glasgow G4 ONR, UK \\ ${ }^{\mathrm{b}}$ Institute of Biomedical and Life Sciences, University of Glasgow, Joseph Black Building, Glasgow G12 8QQ, UK
}

Received 31 August 1999; received in revised form 14 December 1999; accepted 17 January 2000

\begin{abstract}
Drug delivery systems comprising vesicles prepared from one amphiphile encapsulating vesicles prepared from a second amphiphile have not been prepared previously due to a tendency of the bilayer components of the different vesicles to mix during preparation. Recently we have developed polymeric vesicles using the new polymer-palmitoyl glycol chitosan and cholesterol in a 2:1 weight ratio. These polymeric vesicles have now been encapsulated within egg phosphatidylcholine (egg PC), cholesterol (2:1 weight ratio) liposomes yielding a vesicle in vesicle system. The vesicle in vesicle system was visualised by freeze fracture electron microscopy. The mixing of the different bilayer components was studied by monitoring the excimer fluorescence of pyrene-labelled polymeric vesicles after their encapsulation within egg PC liposomes or hexadecyl diglycerol ether niosomes. A minimum degree of lipid mixing was observed with the polymeric vesicle-egg PC liposome system when compared to the polymeric vesicle-hexadecyl diglycerol ether niosome system. The polymeric vesicle-egg PC vesicle in vesicle system was shown to retard the release of encapsulated solutes. $28 \%$ of 5(6)-carboxyfluorescein (CF) encapsulated in the polymeric vesicle compartment of the vesicle in vesicle system was released after $4 \mathrm{~h}$ compared to the release of $62 \%$ of encapsulated $\mathrm{CF}$ from plain polymeric vesicles within the same time period. (C) 2000 Elsevier Science B.V. All rights reserved.
\end{abstract}

Keywords: Liposomes; Phosphatidylcholine; Chitosan; Glycol chitosan; Polymeric vesicles; Multi-component systems; Niosomes

\section{Introduction}

Vesicles such as liposomes, prepared from phospholipids (Gregoriadis, 1993, 1995) and niosomes, prepared from non ionic surfactants (Flo-

\footnotetext{
* Corresponding author.:Fax: + 44-141-5526443.

E-mail address: i.fuchegbu@strath.ac.uk (I.F. Uchegbu)
}

rence, 1993; Uchegbu and Vyas, 1998) have been studied as drug delivery systems for a number of years. There are currently a number of commercially available liposomal formulations which include liposomal doxorubicin (Doxil ${ }^{\mathbb{B}}$ ) (Winterhalter et al., 1997) and daunorubicin $\left(\right.$ DaunoXome $\left.{ }^{\circledR}\right)($ Fox, 1995) for the treatment of Kaposi's sarcoma and also liposomal ampho- 
tericin (Ambisome ${ }^{\circledR}$ ) (Boswell et al., 1998) for the treatment of systemic fungal infections. These formulations are beneficial in targeting drugs to the site of pathology and away from potential sites of toxicity with the drugs usually being encapsulated within unilarnellar vesicles.

The production of multi-component drug delivery systems in which vesicles prepared from one type of amphiphile encapsulate vesicles prepared from a different amphiphile (as shown schematically in Fig. 1a) has not been attempted due to the tendency of the different bilayer components to mix. However solid particulate matter has been encapsulated within vesicles (Antimisiaris et al., 1993; Arrault et al., 1997) and vesicles have been
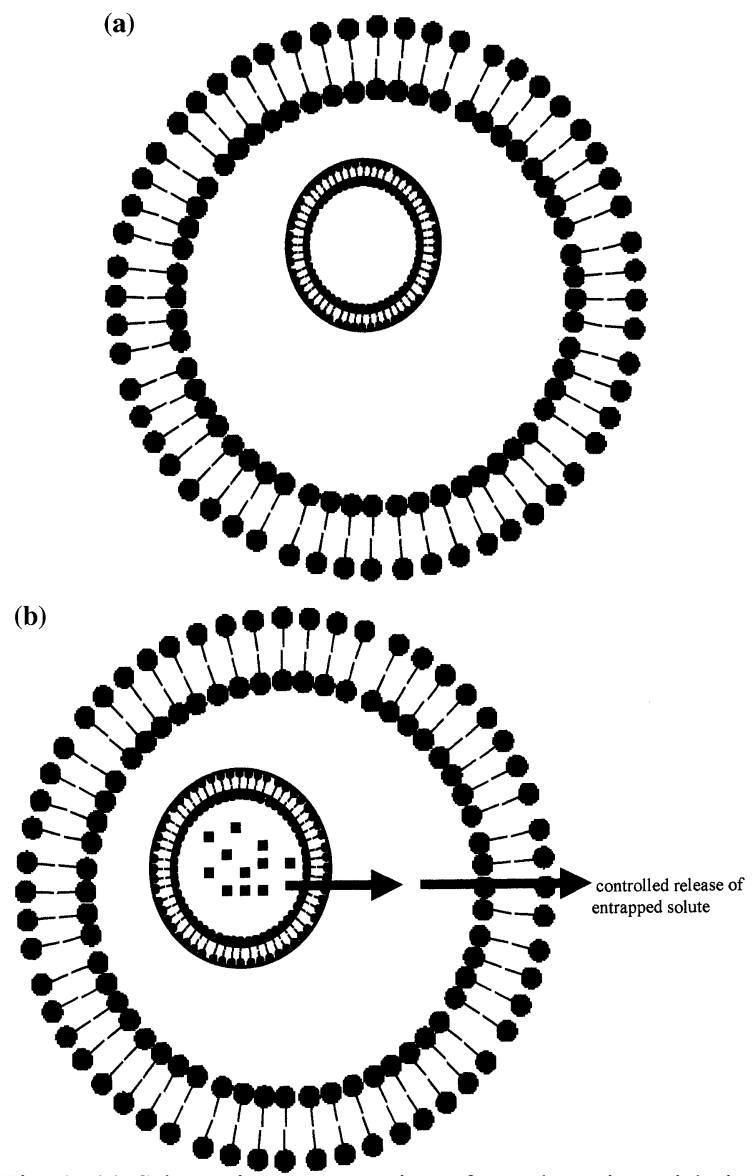

Fig. 1. (a) Schematic representation of a polymeric vesicle in liposome system; (b) schematic representation of the controlled release properties offered by the vesicle in vesicle system. formulated within both water in oil emulsions (Yoshioka and Florence, 1994) and non ionic surfactant based organogels (Murdan et al., 1996). It is envisaged that the encapsulation of a drug/ enzyme/ vaccine/ gene loaded vesicle within a second vesicle will yield a system with improved controlled release properties (Fig. 1b). The fabrication of just such a vesicle in vesicle system using polymeric vesicles is the subject of the current study. Polymeric vesicles (Uchegbu et al., 1998a,b) have been prepared by us from specially designed amphiphilic polymers, the latter of which are synthesised by grafting pendant hydrophobic (Uchegbu et al., 1998b) or hydrophobic and hydrophilic groups (Uchegbu et al., 1998a) on to soluble linear polymers. Polymeric vesicles prepared from palmitoyl glycol chitosan, cholesterol (2:1 weight ratio) (Uchegbu et al., 1998b) have in turn been encapsulated within Egg PC, cholesterol (2:1 weight ratio) liposomes to yield a vesicle in vesicle system.

\section{Materials and methods}

\subsection{Materials}

Palmitoyl glycol chitosan (Fig. 2) was synthesised and characterised as previously described (Uchegbu et al., 1998b). Cholesterol, dicetyl phosphate, phosphatidylcho line, 10(1-pyrene) decanoic acid, 5(6)-carboxyfluorescein and gold chloride were all supplied by Sigma Chemical Co., UK. Dicetyl phosphate was supplied by Fluka Chemical, UK. All organic solvents and phosphate buffered saline tablets were supplied by Merck Chemical Co., UK. Hexadecyl diglycerol ether $\left(\mathrm{C}_{16} \mathrm{G}_{2}\right)$ was a kind gift from L'Oreal, France. Colloidal gold (20nm) was manufactured by sodium citrate reduction of gold chloride (Mey, 1983).

\subsection{Vesicle preparation}

Polymeric vesicles were prepared from palmitoyl glycol chitosan as previously described (Uchegbu et al., 1998b). Egg PC liposomes were prepared by dissolving egg PC (15 mg) and 


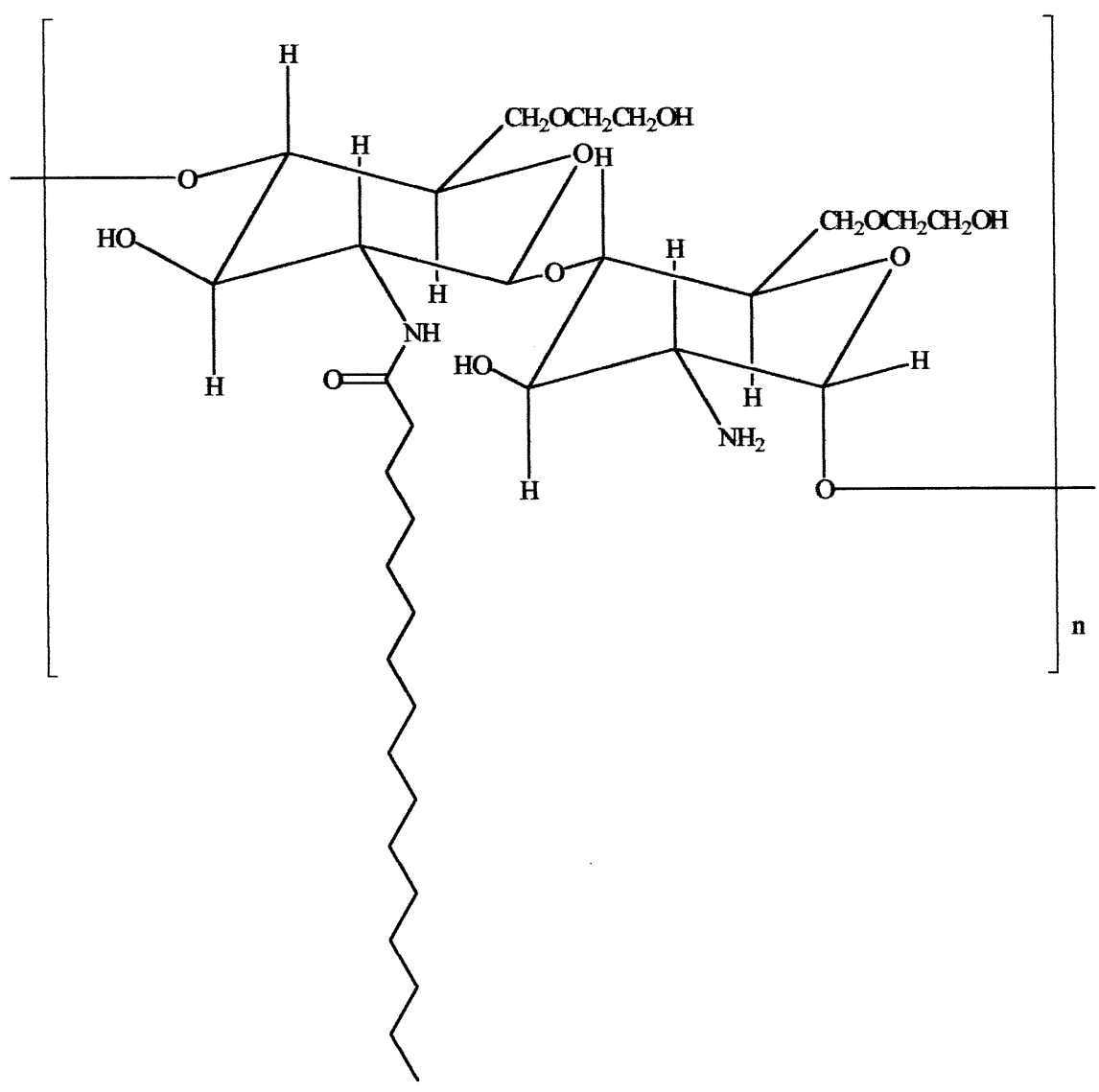

palmitoyl glycol chitosan

Fig. 2. Palmitoyl glycol chitosan.

cholesterol $(7 \mathrm{mg})$ in chloroform $(10 \mathrm{ml})$ and the solution evaporated to dryness under reduced pressure at $40^{\circ} \mathrm{C}$. The resulting thin lipid film was then hydrated with water $(5 \mathrm{ml})$ by shaking for 30 min at RT to yield a homogenous dispersion of egg PC liposomes.

Polyhedral niosomes, so called because they present in an unusual polyhedral shape are prepared without cholesterol and the incorporation of $50 \mathrm{~mole} \%$ cholesterol into the formulation gives rise to the more usual spherical niosomes (Uchegbu et al., 1996, 1997). Polyhedral niosomes were prepared by dissolving $\mathrm{C}_{16} \mathrm{G}_{2}(28 \mathrm{mg})$ and dicetyl phosphate $(1 \mathrm{mg})$ in chloroform $(10 \mathrm{ml})$ and evaporating the solution to dryness under reduced pressure at $40^{\circ} \mathrm{C}$. The resulting thin film of surfactants was hydrated with water $(4 \mathrm{ml})$ by shaking for $\mathrm{I} h$ at $60^{\circ} \mathrm{C}$.

Spherical niosomes were prepared by dissolving $\mathrm{C}_{16} \mathrm{G}_{2} \quad(14 \mathrm{mg})$, cholesterol (14 $\left.\mathrm{mg}\right)$ and dicetylphosphate $(1 \mathrm{mg})$ in chloroform $(10 \mathrm{ml})$ and the solution evaporated to dryness under reduced pressure at $40^{\circ} \mathrm{C}$. The resulting lipid film was hydrated with water by shaking for $\mathrm{I} h$ at $60^{\circ} \mathrm{C}$.

\subsection{Lipid/surfactant mixing experiments}

Pyrene forms excited dimers (excirners) in a concentration dependant manner and the excitation of pyrene excimers at $340 \mathrm{~nm}$ leads to a fluorescent emission at $477 \mathrm{~nm}$, a red shift with 
respect to the emission due to pyrene monomers (392 nm) (Galla and Hartmann, 1980; Pownall and Smith, 1989). Hence by using pyrene tagged lipids, any dilution of pyrene, as would occur on transfer of pyrene lipids from one vesicle bilayer to another, may be monitored by measuring the ratio of pyrene excirner to monomer emission (E/M) (Pownall and Smith, 1989). This is due to the fact that a transfer of the pyrene label from one lipid bilayer to another would lead to a reduction in $\mathrm{E} / \mathrm{M}$. This method was used to study the degree of mixing between the different vesicle formulations that were to be used to prepare the vesicle in vesicle system. The pyrene concentration in compared systems was kept constant.

Pyrene labelled polymeric vesicles were prepared by dissolving palmitoyl glycol chitosan (8 $\mathrm{mg}$ ), cholesterol (4 mg) and 10 (1-pyrene) decanoic acid $(1.5 \mathrm{mg})$ in chloroform $(5 \mathrm{ml})$. The solution was evaporated to dryness under reduced pressure at $40^{\circ} \mathrm{C}$ and the resulting film hydrated with water $(4 \mathrm{ml})$. The dispersion so obtained was then probe sonicated (Soniprobe Instruments) for $2 \times 2 \mathrm{~min}$ with the instrument set at $20 \%$ of its maximum output.

Zero point four milliliters of pyrene labelled polymeric vesicles were added to isopropanol $(3.6 \mathrm{ml})$ to produce a dispersion giving $100 \%$ monomer emission and to water $(3.6 \mathrm{ml})$ to produce a dispersion giving $100 \%$ excimer emission. The emission spectra $(350-550 \mathrm{~nm})$ of these mixtures were recorded on a Perkin-Elmer LS-50B scanning fluorimeter (excitation $340 \mathrm{~nm}$ ) at 10 min intervals for $1 \mathrm{~h}$ and the $\mathrm{E} / \mathrm{M}$ value computed. The $\mathrm{E} / \mathrm{M}$ ratio remained fairly constant over the $1 \mathrm{~h}$ time period.

Zero point four milliliters of pyrene labelled polymeric vesicles were also added to an aliquot of unlabelled polymeric vesicles $(1.2 \mathrm{ml})$, unlabelled polyhedral niosomes $(1.2 \mathrm{ml})$ or unlabelled spherical niosomes $(1.2 \mathrm{ml})$ and the emission spectra and $\mathrm{E} / \mathrm{M}$ value recorded as described above.

The level of lipid mixing occurring during the preparation of the vesicle in vesicle system was assessed in the following way. A thin film of surfactants/ lipids was prepared as described above using $\mathrm{C}_{16} \mathrm{G}_{2}(28 \mathrm{mg})$ and dicetyl phosphate $(1 \mathrm{mg})$ to give polyhedral niosomes or $\mathrm{C}_{16} \mathrm{G}_{2}(14 \mathrm{mg})$, cholesterol (14 mg) and dicetyl phosphate $(2 \mathrm{mg})$ to give spherical niosomes. The film was hydrated by shaking for $1 \mathrm{~h}$ at $60^{\circ} \mathrm{C}$ with a dispersion $(4 \mathrm{ml})$ of pyrene labelled polymeric vesicles consisting of palmitoyl glycol chitosan $\left(0.5 \mathrm{mg} \cdot \mathrm{ml}^{-1}\right)$, cholesterol $\left(0.25 \mathrm{mg} \cdot \mathrm{ml}^{-1}\right)$ and 10-(1 -pyrene)decanoic acid $(0.25 \mathrm{mM})$. The $\mathrm{E} / \mathrm{M}$ value was once again recorded as detailed above. In a similar manner a thin film prepared from egg PC (1 $5 \mathrm{mg})$ and cholesterol $(7 \mathrm{mg})$ to give egg PC liposomes was hydrated by shaking for $1 \mathrm{~h}$ at $\mathrm{RT}$ with pyrene labelled polymeric vesicles $(4 \mathrm{ml})$ and the $\mathrm{E} / \mathrm{M}$ value once again recorded.

\subsection{Preparation of gold labelled polymeric vesicles}

A colloidal gold dispersion $(20 \mathrm{~nm}, 6 \mathrm{ml})$ was concentrated by centrifugation $(9000 \mathrm{~g} \times 30 \mathrm{~min}$, Beckman L8-55M ultracentrifuge). The clear supernatant was removed and discarded and the colloidal gold collected and diluted to $2.5 \mathrm{ml}$, with distilled water. To this colloidal gold dispersion was then added palmitoyl glycol chitosan (5 $\mathrm{mg}$ ) and cholesterol (2 mg) and the entire mixture probe sonicated as detailed above. To separate unencapsulated gold from encapsulated gold the mixture was further centrifuged $(2200 g \times 5$ min) and the pelletted unencapsulated colloidal gold discarded. Gold-labelled polymeric vesicles were visualised by electron microscopy as detailed below.

\subsection{Preparation of the vesicle in vesicle system - encapsulation of gold labelled polymeric vesicles within egg PC liposomes}

A thin film of egg PC (8 mg) and cholesterol (4 mg) was hydrated with the polymeric vesicle suspension $(2.5 \mathrm{ml})$ encapsulating colloidal gold by shaking the mixture for $10 \mathrm{~min}$ at $\mathrm{RT}$. In some experiments polymeric vesicles encapsulating colloidal gold in which the unencapsulated colloidal gold had not been removed were used to prepare the vesicle in vesicle system. 


\subsection{Electron microscopy}

Freeze fracture electron microscopy was used to visualise both the gold-labelled polymeric vesicles, the gold-labelled vesicle in vesicle system and egg PC liposomes. Specimens were fast-frozen by plunging into liquid propane/ isopentane at $180^{\circ} \mathrm{C}$ and transferred cold into a high vacuum freeze-fracture system. Specimens were fractured with a Balzars complementary replica device at $-100^{\circ} \mathrm{C}$ and the exposed faces replicated with platinum carbon at $45^{\circ}$ and backed by carbon at $90^{\circ}$. Replicas were cleaned in acetone/ alcohol, rinsed in distilled water, dried on Formvar-coated grids and finally imaged using a LEO 902 energyfiltering electron microscope.

Transmission electron microscopy with negative staining was also used to visualise gold-labelled polymeric vesicles. Droplets of vesicle preparation were mixed in equal $(20 \mu \mathrm{l})$ volumes with $1 \%$ uranyl formate in distilled water $(\mathrm{pH}, 4.8)$ on a specimen support grid and immediately dried down using filter paper. The negatively stained grid samples were imaged in a LEO 902 energy filtering electron microscope at $8 \mathrm{kV}$.

\subsection{CF loaded vesicle in vesicle system}

CF loaded polymeric vesicles were prepared by sonicating a dispersion of palmitoyl glycol chitosan $(16 \mathrm{mg})$ and cholesterol $(8 \mathrm{mg})$ with a solution of CF (2.2 mM, $8 \mathrm{ml})$. Separation of unentrapped $\mathrm{CF}$ was achieved by ultracentrifugation $(150000 \mathrm{~g} \times 30 \mathrm{~min})$ and the supernatant discarded. The pelleted CF loaded vesicles were resuspended in $5 \mathrm{ml}$ phosphate buffered saline (PBS, pH, 7.4). The CF loaded vesicle in vesicle system was prepared by hydrating a thin film of Egg PC (15 mg) and cholesterol $(7 \mathrm{mg})$, with the $\mathrm{CF}$ loaded polymeric vesicle dispersion $(5 \mathrm{ml})$. Hydration of the phospholipid cholesterol film was achieved by shaking at RT for $1 \mathrm{~h}$. Unencapsulated CF loaded polymeric vesicles were separated by centrifugation $(1400 \mathrm{~g} \times 30 \mathrm{~min})$ and the pelleted vesicle in vesicle system resuspended in PBS $(8 \mathrm{ml})$.

CF loaded egg PC liposomes were prepared by hydrating an egg PC (15 mg) and cholesterol (7 $\mathrm{mg}$ ) film with $5 \mathrm{ml}$, CF $(2.2 \mathrm{mM})$. This was carried out by shaking for $10 \mathrm{~min}$ at RT. The assay for encapsulated CF was carried out as previously described (Uchegbu et al., 1992).

The release of $\mathrm{CF}$ from (a) the vesicle in vesicle system; (b) Egg PC liposomes; and (c) polymeric vesicles was also studied. The three vesicle preparations were normalised for $\mathrm{CF}$ concentration and a control sample of CF solution also prepared. 1 $\mathrm{ml}$, aliquots of each of the vesicle formulations and the control CF solution were placed in $10 \mathrm{~cm}$ lengths of Visking dialysis tubing ( $\mathrm{Mw}$ cut off $12000-14000$ ) and both ends sealed. These were then placed in $20 \mathrm{ml}$, PBS $(\mathrm{pH}, 7.4)$ and the dialysate sampled at various time intervals. Released $\mathrm{CF}$ was quantified by fluorimetric analysis (excitation, $486 \mathrm{~nm}$, emission, $514 \mathrm{~nm}$ ).

\section{Results}

\subsection{Lipid/surfactant mixing experiments}

On hydration of a lipid/surfactant film with the polymeric vesicle formulation, mixing of the bilayer constituents would lead not to a vesicle in vesicle system as desired, but a vesicle formulation in which the bilayers were made up of mixtures of lipids arising from both formulations. Hence an assessment of the degree of mixing of both vesicles comprising the vesicle in vesicle system was deemed necessary.

The presence of the peak at $477 \mathrm{~nm}$ in pyrene labelled polymeric vesicles (Fig. 3a) is indicative of the presence of pyrene excimers, while the presence of the peak at $392 \mathrm{~nm}$ (Fig. 3b) when the labelled polymeric vesicles are disrupted with isopropanol is indicative of the presence of pyrene monomers (Viani et al., 1988). It is clear from the spectra (Fig. 3a and b) and respective $\mathrm{E} / \mathrm{M}$ values (Table 1) that the pyrene label was effectively incorporated into the polymeric vesicle bilayer. The formation of monomers on disruption of the bilayer with isopropanol indicates that the pyrene label was originally incorporated into the polymeric vesicle bilayer in discrete domains of local high concentration. Once the bilayer was disrupted by the solubilisation of cholesterol with 
a.
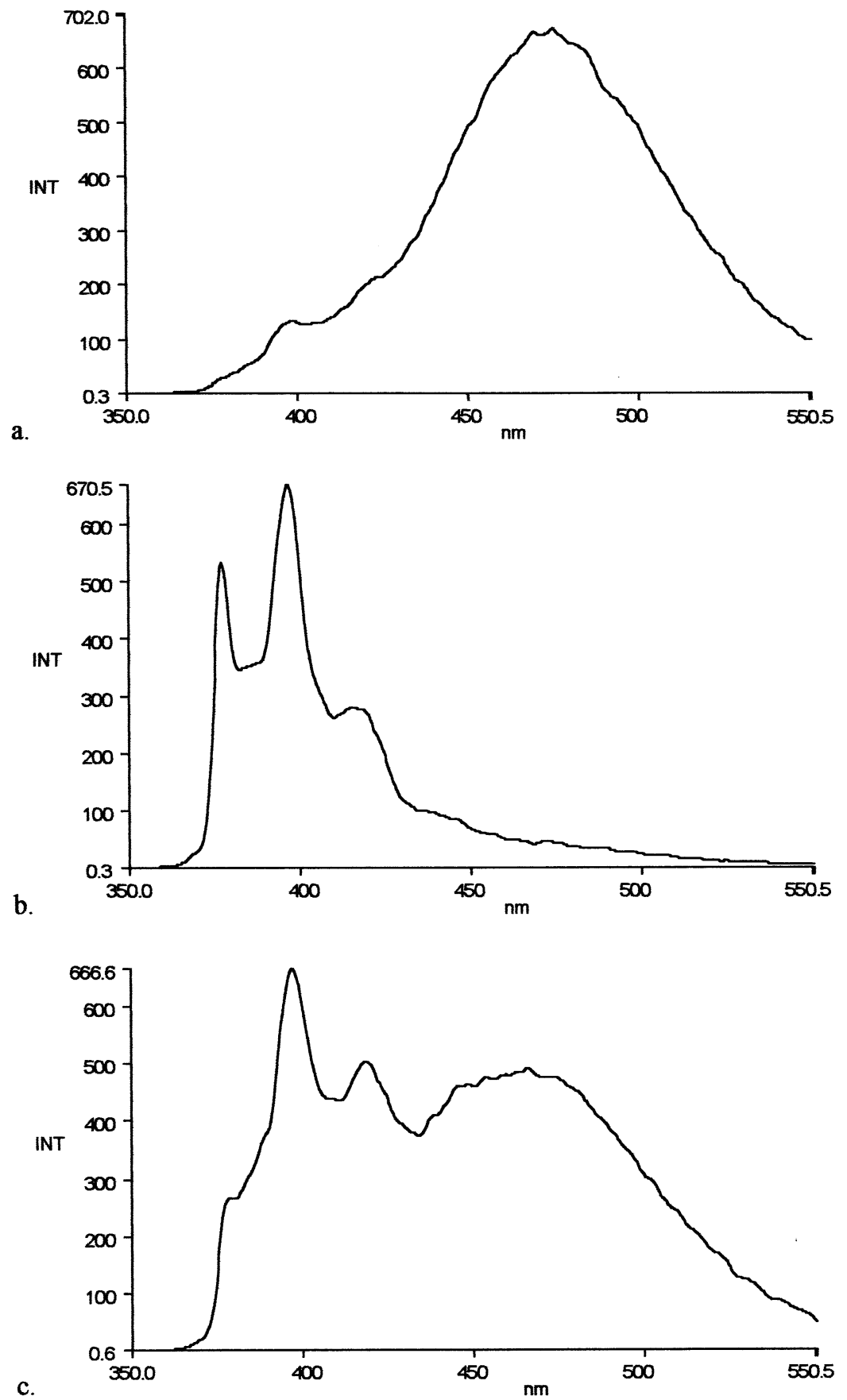

Fig. 3. Emission spectra of pyrene labelled polymeric vesicle systems (excitation, $340 \mathrm{~nm}$ ) a) in water (pyrene concentration, 0.1 $\mathrm{mM}$ ); (b) disrupted with nine volumes of isopropanol (pyrene concentration $0.1 \mathrm{mM}$ ), c) incubated for $1 \mathrm{~h}$ with unlabelled polymeric vesicles (pyrene concentration $0.25 \mathrm{mM}$ ); (d) incubated for $1 \mathrm{~h}$ with unlabelled polyhedral niosomes (pyrene concentration, 0.25 $\mathrm{mM}$ ). Emission spectra (excitation, $340 \mathrm{~nm}$ ) of (e) a polyhedral niosome suspension prepared in the presence of pyrene labelled polymeric vesicles; and (f) an egg PC liposome suspension prepared in the presence of pyrene labelled polymeric vesicles. 

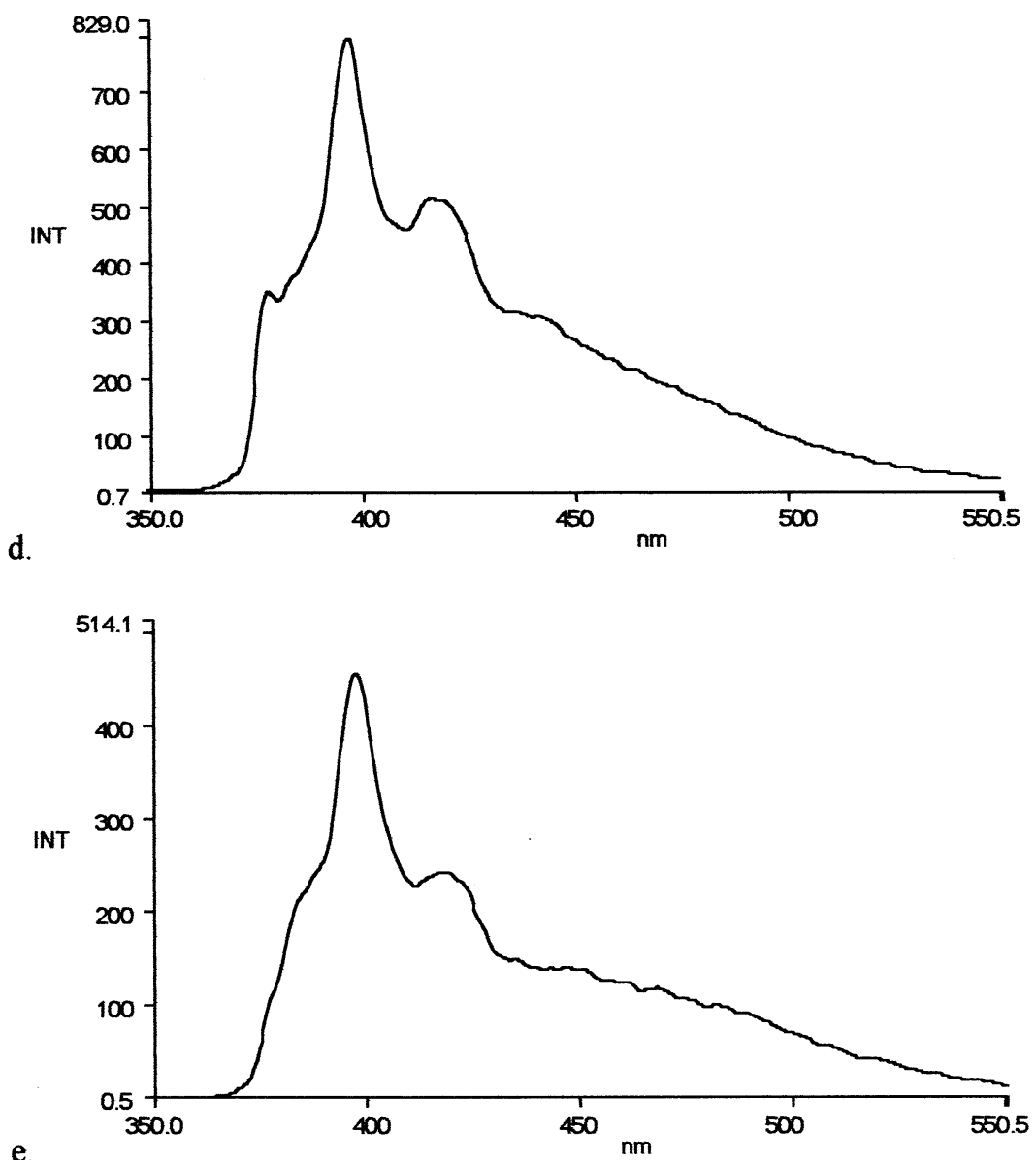

e.

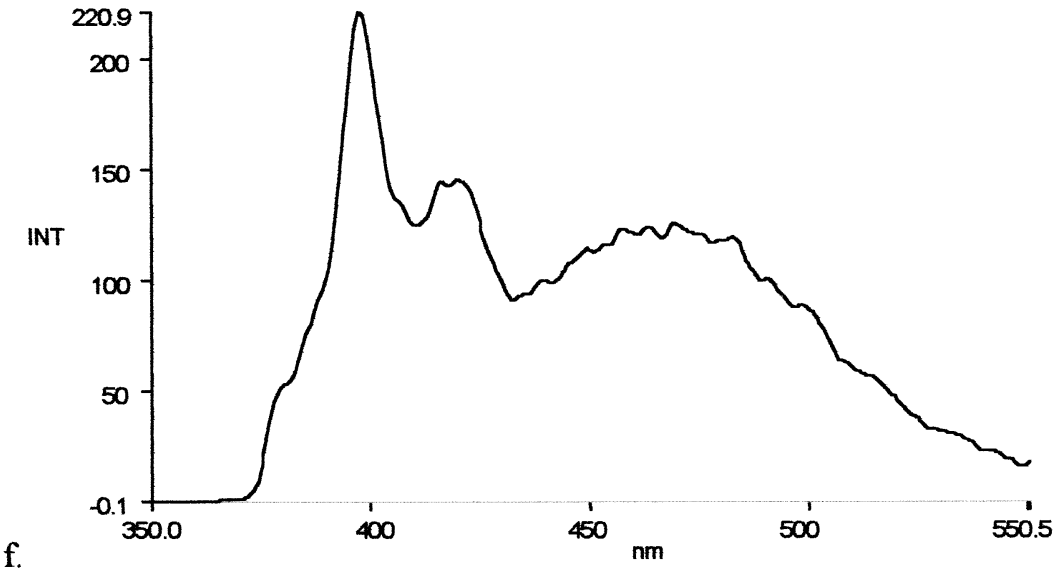

Fig. 3. (Continued) 
Table 1

$\mathrm{E} / \mathrm{M}$ values of pyrene labelled polymeric vesicles incubated for $1 \mathrm{~h}$ in various media

\begin{tabular}{|c|c|c|c|}
\hline Incubation media & $\mathrm{E} / \mathrm{M}$ value & $\begin{array}{l}\text { 10-(1-Pyrene)decanoic acid } \\
\text { concentration }(\mathrm{mM})\end{array}$ & $\begin{array}{l}\text { Final concentration of } \\
\text { surfactants/lipids }\left(\mathrm{mg} \cdot \mathrm{ml}^{-1}\right)\end{array}$ \\
\hline Water & 4.93 & $0.1 \mathrm{mM}$ & 0.3 \\
\hline Isopropanol & 0.06 & $0.1 \mathrm{mM}$ & 0.3 \\
\hline $\begin{array}{l}\text { Unlabelled polymeric } \\
\text { vesicles }\end{array}$ & 0.73 & $0.25 \mathrm{mM}$ & 5 \\
\hline $\begin{array}{l}\text { Unlabelled polyhe- } \\
\text { dral niosomes }\end{array}$ & 0.19 & $0.25 \mathrm{mM}$ & 5.3 \\
\hline $\begin{array}{l}\text { Unlabelled spherical } \\
\text { niosomes }\end{array}$ & 0.16 & $0.25 \mathrm{mM}$ & 5 \\
\hline
\end{tabular}

isopropanol these discrete domains were destroyed and hence only the monomer peak was seen (Fig. 3b, Table 1).

The transfer of the pyrene label from polymeric vesicle bilayers to non labelled vesicles and hence the degree of lipid/surfactant mixing was assessed by incubating pyrene labelled polymeric vesicles with unlabelled niosomes. The level of lipid/surfactant mixing was found to be lowest when labelled polymeric vesicles were incubated with unlabelled polymeric vesicles (Fig. 3c, Table 1) but highest when labelled polymeric vesicles were incubated with polyhedral or spherical niosomes (Fig. 3d, Table 1). On preparing polyhedral niosomes, spherical niosomes and egg PC liposomes in the presence of pyrene labelled polymeric vesicles, the level of lipid/surfactant mixing as determined by the fall in $\mathrm{E} / \mathrm{M}$ values followed the trend egg $\mathrm{PC}<$ spherical niosomes $<$ polyhedral niosomes (Fig. $3 \mathrm{e}$ and $\mathrm{f}$, Table 2). There was obviously some transfer of the pyrene label when labelled vesicles were incu- bated with unlabelled vesicles but this was minimal in two notable cases: (a) when labelled vesicles were incubated with unlabelled polymeric vesicles; and (b) when labelled polymeric vesicles were used to hydrate an egg PC-cholesterol film.

\subsection{Imaging of the vesicle in vesicle system}

Colloidal gold effectively labelled the polymeric vesicles (Fig. 4a and b) and the presence of gold labelled polymeric vesicles encapsulated within larger Egg PC vesicles is shown in Fig. 4(c). Plain egg PC liposomes are shown in Fig. 4(d). Freeze fracture electron microscopy was chosen as the imaging technique for the vesicle in vesicle system since the fracturing of vesicles during sample preparation is able to produce images of the vesicle interior and hence clearly show vesicles contained within vesicles. However to obtain useful images both internal and external vesicles require to be fractured simultaneously.

Table 2

$\mathrm{E} / \mathrm{M}$ values of unlabelled formulations prepared in the presence of pyrene labelled polymeric vesicles

\begin{tabular}{llll}
\hline Formulation & E/M value & $\begin{array}{l}10 \text {-(I-Pyrene)decanoic acid } \\
\text { concentration }(\mathrm{mM})\end{array}$ & $\begin{array}{l}\text { Final concentration of } \\
\text { surfactants } / \text { lipids }\left(\mathrm{mg} \cdot \mathrm{ml}{ }^{-1}\right)\end{array}$ \\
\hline Polyhedral niosomes & 0.26 & $0.25 \mathrm{mM}$ & 8.0 \\
Spherical niosomes & 0.44 & $0.25 \mathrm{mM}$ & 8.0 \\
Egg PC liposomes & 0.57 & $0.25 \mathrm{mM}$ & 6.0 \\
\hline
\end{tabular}


It is theoretically possible that any free unencapsulated gold may become entrapped within egg PC liposomes during their formation. However, it is more probable that the image obtained in Fig. 4(c) represents the gold labelled polymeric vesicles entrapped within phosphatidylcholine vesicles as this image shows a vesicle entrapped within a vesicle and not simply a vesicle entrapping free gold.

\section{3. $C F$ release}

As these vesicle in vesicle systems are being developed for the controlled release of bioactive substances, the release of a model compound the aqueous soluble fluorophore- $\mathrm{CF}$ from the vesicle in vesicle system was studied (Fig. 5). Polymeric vesicles released $62 \%$ of encapsulated $\mathrm{CF}$ after $4 \mathrm{~h}$ compared to the release of $28 \%$ encapsulated $\mathrm{CF}$

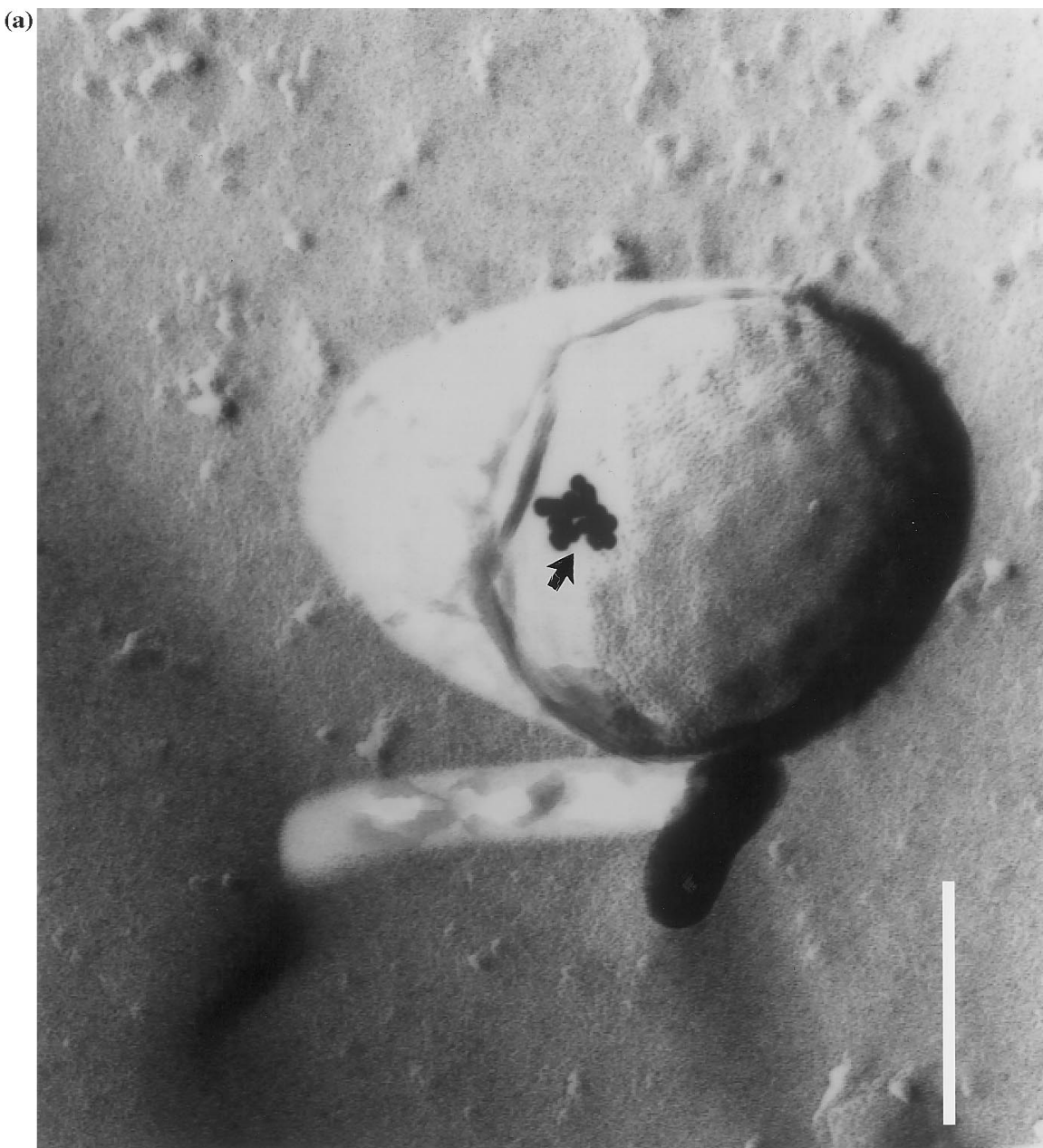

Fig. 4. (a) Freeze fracture electron micrograph of gold $(20 \mathrm{~nm})$ labelled polymeric vesicles, gold particles are indicated by arrow, bar, $250 \mathrm{~nm}$; (b) Transmission electron micrograph with negative staining of gold $(20 \mathrm{~nm})$ labelled polymeric vesicles, gold particles are indicated by arrows, bar, $400 \mathrm{~nm}$; (c) Freeze fracture electron micrograph of large egg PC liposome encapsulating polymeric gold $(20 \mathrm{~nm}$ ) labelled vesicle, gold particles are indicated by arrow, bar, $400 \mathrm{~nm}$; (d) Freeze fracture electron micrograph of egg PC liposomes, bar, $600 \mathrm{~nm}$. 


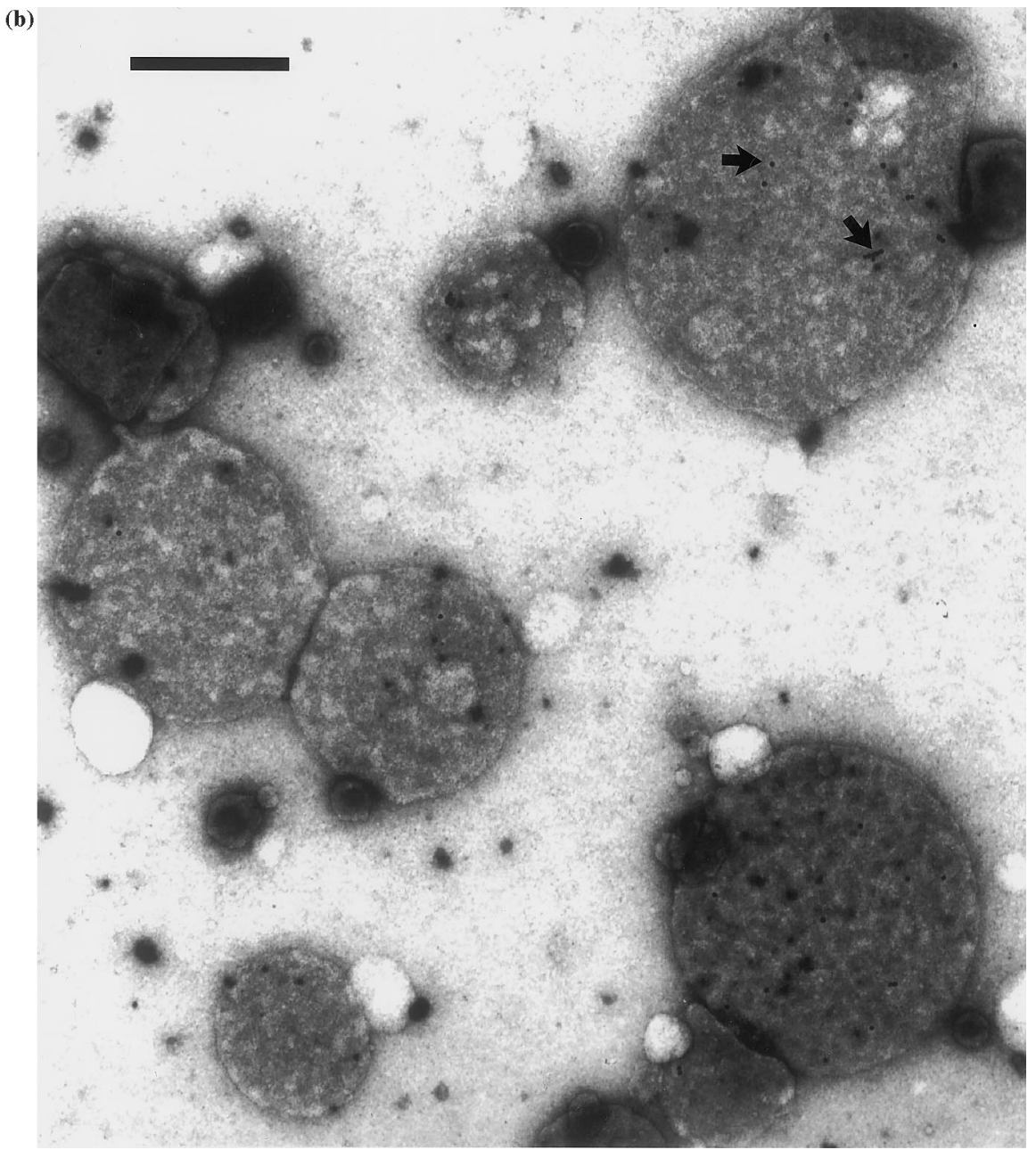

Fig. 4. (Continued)

from the vesicle in vesicle system over the same time period, evidence that the vesicle in vesicle system retards the release of encapsulated solutes. Egg PC liposomes released $37 \%$ of encapsulated $\mathrm{CF}$ under similar conditions. The difference in the release rate of $\mathrm{CF}$ when $\mathrm{CF}$ loaded polymeric vesicles are compared to the sample in which $\mathrm{CF}$ loaded polymeric vesicles were used to hydrate a film of egg PC-cholesterol (Fig. 5) is indicative of the polymeric vesicles being entrapped within the egg PC liposomes.

\section{Discussion}

When taken collectively and not in isolation the experimental data from each set of experiments point to the fact that a vesicle in vesicle system may be fabricated from polymeric vesicles and egg PC liposomes. This work represents the first time that vesicle prepared from one amphiphile have been encapsulated within vesicles prepared from a second amphiphile. Lipid/surfactant mixing experiments were employed to select the formula- 
tion to be used to prepare the multicomponent system and the formation of the vesicle in vesicle system confirmed by electron microscopy.

Egg PC is an ideal material for formulation of the outer component of the vesicle in vesicle system by virtue of the low gel to liquid phase transition temperature of this phospholipid (New, 1990). Hence vesicles may be prepared by film hydration at room temperature (Fig. 4d) and once formed are quite stable at room temperatue (RT). Bilayer phospholipids are known to be more mobile at elevated temperatures (Israelachvili, 1992) and as such elevated temperatures during preparation of the vesicle in vesicle system would be more likely to result in perturbation of the polymeric vesicle membrane by phospholipid molecules. Preparation of egg PC vesicles in the presence of polymeric vesicles at RT would also limit the mobility in the polymeric vesicle bilayer and once again reduce the likelihood of intervesicular bilayer mixing.

The preparation of both cholesterol containing formulations, i.e. the spherical niosomes and the egg PC liposomes in the presence of pyrene labelled polymeric vesicles revealed a greater degree of lipid mixing in the case of the spherical niosomes than the egg PC liposomes (Table 2). It is possible that this could be due to the lower level

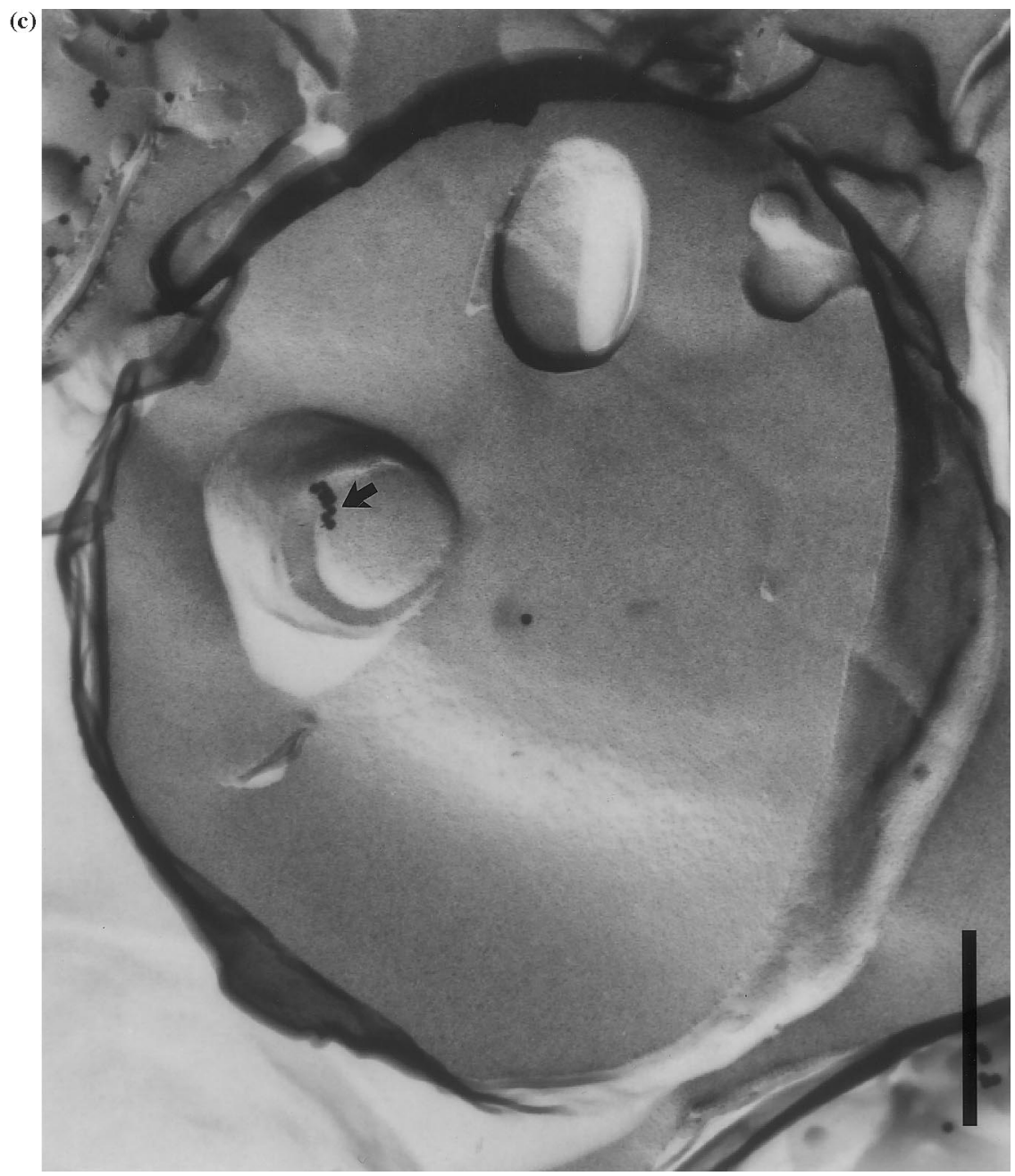

Fig. 4. (Continued) 


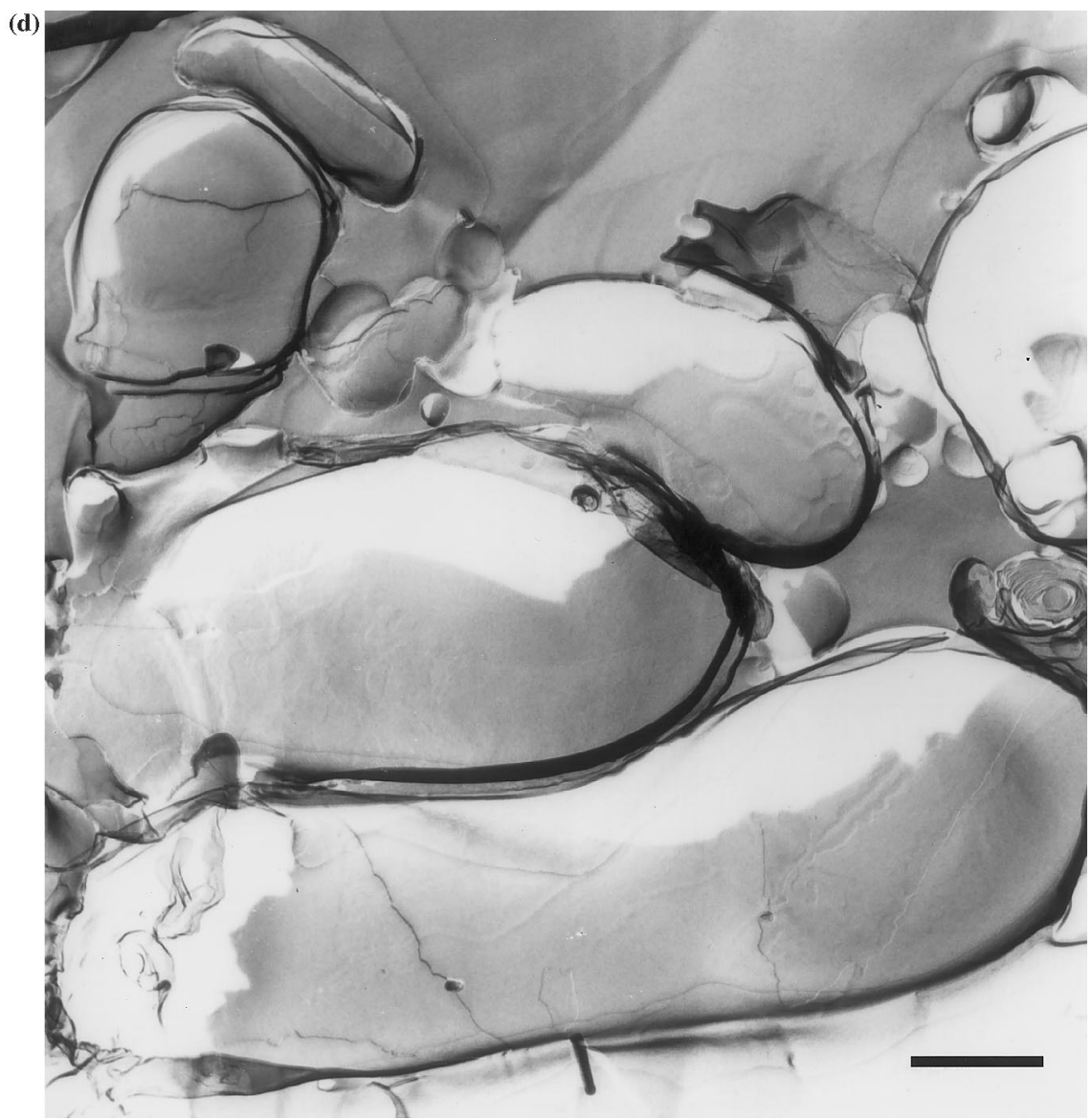

Fig. 4. (Continued)

of overall lipid/surfactant in the egg PC system. This is unlikely however, as the lipid mixing in the polyhedral and spherical niosome systems is further enhanced at lower overall lipid concentrations (Table 1). The lower degree of lipid mixing in the egg PC system could also be due to the molecular differences between $\mathrm{C}_{16} \mathrm{G}_{2}$ and egg PC. $\mathrm{C}_{16} \mathrm{G}_{2}$ is a single tailed amphiphile while egg PC consists of a mixture of largely double tailed amphiphiles (Fig. 6). The inter-bilayer transfer of the double tailed amphiphile would be a higher energy process than the transfer of the single tailed amphiphile since this transfer will involve a crossing of the external aqueous layer (Pownall and Smith, 1989). Hence perturbation of the polymeric vesicle bilayer by phospholipid molecules with the resultant inter-vesicular bilayer mixing would occur less readily with the egg PC system when compared to the $\mathrm{C}_{16} \mathrm{G}_{2}$ system. However the essential conclusion to be drawn for the data in Table 2 is that egg PC liposomes appear to be a

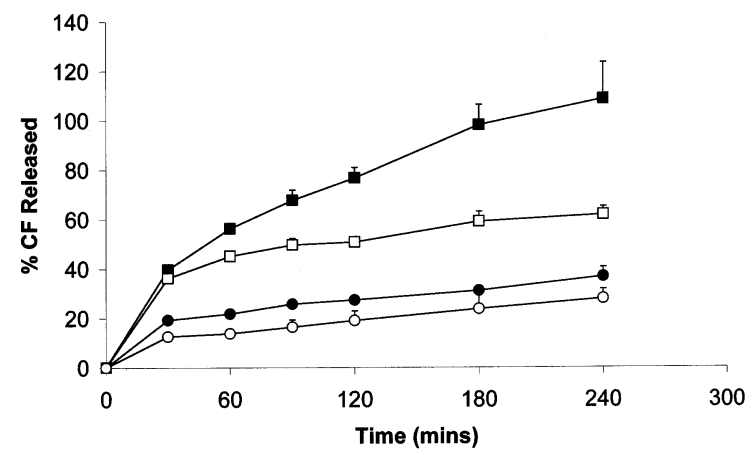

Fig. 5. The release of $\mathrm{CF}(0.03 \mathrm{mM})$ from $(\bigcirc)$, vesicle in vesicle system (polymeric vesicles encapsulated in egg PC liposomes), ๑, egg PC liposomes; $\square$, polymeric vesicles; $\square$, $\mathrm{CF}$ solution. 


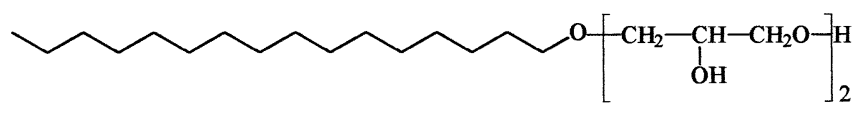

a

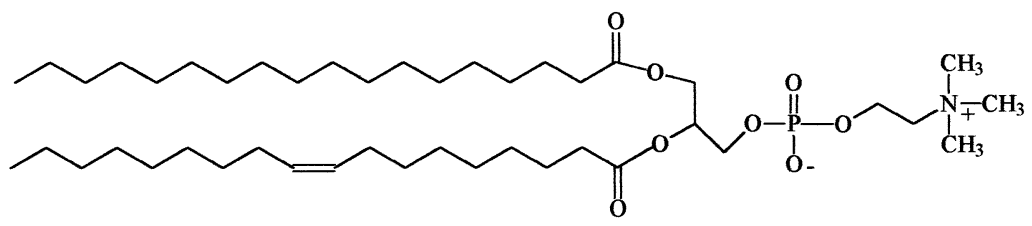

b

Fig. 6. Structural formulae for (a) $\mathrm{C}_{16} \mathrm{G}_{2}$; and (b) a typical phospholipid component of egg PC.

better choice for the external vesicles than any of the $\mathrm{C}_{16} \mathrm{G}_{2}$ systems.

A comparison of the level of lipid/surfactant mixing observed when pyrene labelled polymeric vesicles were incubated with either unlabelled polymeric vesicles or unlabelled spherical niosomes, both of which contain cholesterol at a level of $33 \% \mathrm{w} / \mathrm{w}$, revealed less lipid/surfactant mixing in the case of the polymeric bilayers (Table 1). This suggests that the polymeric bilayer is a less mobile environment when compared to the spherical niosome bilayer.

The formation of a vesicle in vesicle system (Fig. 4c) provides a greater control of the release rate of encapsulated solutes as shown in Fig. 5 due to the fact that the aqueous solute has to cross at least two well defined membrane barriers (Fig. 1b). This can be used to provide controlled release immunological adjuvants or anti-cancer formulations. However more importantly the vesicle in vesicle system may be used to incorporate two different active ingredients as for example in combination chemotherapy or the for the incorporation of an enzyme within one compartment and a prodrug within a second compartment for site specific activation. These applications will be explored in subsequent studies.

\section{Acknowledgements}

This work was funded by the Wellcome Trust by way of a Sir Henry Wellcome Commemorative Award for Innovative Research.

\section{References}

Antimisiaris, S.G., Jayasekera, P., Gregoriadis, G., 1993. Liposomes as vaccine carriers. J. Immun. Meth. 166, $271-$ 280 .

Arrault, J., Grand, C., Poon, W.C.K., Cates, M.E., 1997. Stuffed onions: particles in multilamellar vesicles. Europhys. Lett. 38, 625-630.

Boswell, G.W., Buell, D., Bekersky, I., 1998. AmBisome (liposomal amphotericin 13): a comparative review. J. Clin. Pharmacol. 38, 583-592.

Florence, A.T., 1993. Non ionic surfactant vesicles; preparation and characterisation. In: Gregoriadis, G. (Ed.), Liposome Technology, vol. 2. CRC Press, Boca Raton, pp. $157-176$.

Fox, J.L., 1995. FDA advisers okay Nexstar's daunoxome. Bio-Technology 13, 635-636.

Galla, H.J., Hartmann, W., 1980. Excimer-forming lipids in membrane research. Chem. Phys. Lip. 27, 199-219.

Gregoriadis, G., 1993. Liposome Technology. CRC Press, Boca Raton, FL.

Gregoriadis, G., 1995. Engineering liposomes for drug delivery: progress and problems. Tibtech 13, 527-536.

Israelachvili, J., 1992. Intermolecular And Surface Forces. Academic Press, London. 
Mey, J.D., 1983. Colloidal gold probes in immunocytochemistry. In: Polack, J.M., Noorden, S.V. (Eds.), Immunocytochemistry: Practical Applications in Pathology and Biology. Wright/PSG, Bristol, pp. 82-112.

Murdan, S., Gregoriadis, G., Florence, A.T., 1996. Non ionic surfactant based organogels incorporating niosomes. STP Pharma. Sci. 6, 44-48.

New, R.R.C., 1990. Introduction. In: New, R.R.C. (Ed.), Liposomes a Practical Approach. Oxford University Press, Oxford, pp. 1-32.

Pownall, H.J., Smith, L.C., 1989. Pyrene-labelled lipids: versatile probes of membrane dynamics in vitro and in living cells. Chem. Phys. Lip. 50, 191-211.

Uchegbu, I.F., Bouwstra, J.A., Florence, A.T., 1992. Large disk-shaped structures (discomes) in non ionic surfactant vesicle to micelle transitions. J. Phys. Chem. 96, 1054810553.

Uchegbu, I.F., McCarthy, D., Sch5Wein, A., Florence, A.T., 1996. Phase-transitions in aqueous dispersions of the hexadecyl diglycerol ether (Q16)G(2)) Non ionic surfactant, cholesterol and cholesteryl poly-24-oxyethylene ether-vesicles, tubules, discomes and micelles. STP Pharma. Sci. 6, $33-43$.
Uchegbu, I.R, Schatzlein, A., Vanlerberghe, G., Morgatini, N., Florence, A.T., 1997. Polyhedral non ionic surfactant vesicles. J. Pharm. Pharmacol. 49, 606-610.

Uchegbu, I.F., Schdtzlein, A.G., Tetley, L., Brown, M., Siddique, S., Jack, V., 1998a. Polymeric vesicles from amino acid homopolymers. Proc. Infl. Symp. Control Rel. Bioact. Mater. 25, 186-187.

Uchegbu, I.F., Schdtzlein, A.G., Tetley, L., et al., 1998b. Polymeric chitosan based vesicles for drug delivery. J. Pharm. Pharmacol. 50, 453-458.

Uchegbu, I.F., Vyas, S.P., 1998. Non ionic surfactant vesicles (niosomes) in drug delivery. Int. J. Pharm. 172, 33-70.

Viani, P., Galimberti, C., Marchesini, S., Cervato, G., Cestaro, B., 1988. $N$-pyrene dodecanoyl suflatide as membrane probe: a study of glycolipid behaviour in model membranes. Chem. Phys. Lipids 46, 89-97.

Winterhalter, M., Frederik, P.M., Vallner, J.I, Lasic, D.D., 1997. Stealth $(R)$ liposomes: From theory to product. Adv Drug Del Rev 24, 165-177.

Yoshioka, T., Florence, A.T., 1994. Vesicle (niosome)-in-water-in-oil $(\mathrm{V} / \mathrm{W} / 0)$ emulsions-an in-vitro study. Int. J. Pharm. 108, 117-123. 